\title{
Cancer: Disease Caused by Alteration of Genes
}

\author{
Manzoor Ahmad Malik ${ }^{1 *}$, Zahra Sepehri' ${ }^{2}$, Zafar Amin Shah ${ }^{1}$ \\ ${ }^{1}$ Cancer Diagnostic and Research Center, Department of Immunology and Molecular Medicine, Sher-I-Kashmir Institute of \\ Medical Sciences (SKIMS), Soura, Srinagar, Kashmir-190011, J\&K, India \\ ${ }^{2}$ Department of Internal Medicine, Zabol University of Medical Sciences, Zabol, Iran
}

\section{*Correspondence to Manzoor Ahmad Malik; Email: maliksgpgi@gmail.com}

Received July 23, 2016 Revised August 16, 2016 Accepted August 30, 2016 Published online September 26, 2016

Please cite this article as follows: Malik MA, Sepehri Z, Shah ZA. Cancer: disease caused by alteration of genes. Int J Basic Sci Med. 2016;1(2):3739. doi: $10.15171 /$ ijbms.2016.09.

\begin{abstract}
In normal cells when genetic defects occur, there are some sensitive mechanisms through which the defect might be repaired or apoptosis might be induced. However in tumor cells such mechanisms are not highly sensitive; therefore they cannot detect such abnormalities. This failure in detection and/or repair of the genetic abnormality results in the survival of abnormal cells. This survival is followed up with growth of the cells without normal control and ultimately leads to the rise of malignant clones in the body. Pathways in which these malignant transformations occur, are increasingly being studied to find effective treatments for different carcinomas. Keywords: Cancer, Tumor suppressor genes, Oncogenes and low penetrance genes.
\end{abstract}

Cancer

Growth and division of an individual cell is highly regulated. A cell turns tumourigenic when control over division is lost. Immortalization, transformation, and metastasis are the changes that occur when cell becomes cancerous. The continuous changes result in unrestrained growth and interfere with the normal function of body. Although some cancer types are hereditary, some errors in DNA replication or exposure to carcinogen result in mutation in some somatic cells which can later turn cancerous. Tumors are classified behaviorally into benign and malignant. Benign tumors are slowly growing expansive masses enclosed within a fibrous capsule, whereas malignant tumors are usually rapidly growing, spread into not only local tissue but distant sites, too. The most practical classification of tumors is based on the tissue of origin and type of cell involved; for example, carcinoma is a cancer derived from epithelium, which is the most familiar cancer and more than $90 \%$ of tumors are originated from this tissue. Similarly, sarcoma arises from connective tissue whereas, leukemia arises from bone marrow. The histogenetic classification of benign and malignant tumors is shown in Table $1 .^{1}$
Tumor growth is initiated in the cell in which new mutation results in replication advantage over other cells and the clone derived from this cell undergoes sequential rounds of clonal expansion at exponential rates. Series of subsequent mutations lead to the development of variety of gene products required for the development of malignant phenotype. Carcinogenesis is a multi-step process including initiation, promotion, and progression where normal epithelial tissues are changed to subsequent-hyperplasia to dysplasia to carcinoma in situ, to invasive carcinoma and ultimately to metastatic carcinoma. ${ }^{2}$

\section{Types of Cancers}

Hereditary (or inherited): Every cell (somatic + germline) in the body of a person has a mutation in some highly penetrant genes. This is relatively rare and accounts up to about $5 \%-10 \%$ of all solid tumors and a smaller proportion of hematological malignancies.

Sporadic: Mutations only in somatic cells, comprising more than $90 \%$ of all cancer cases.

Familial cancer: These cancers are caused by both environmental factors and variations in several low penetrance genes. They account for $10 \%-30 \%$ of all cancers,

\footnotetext{
Copyright (C) 2016 The Author(s); Published by Zabol University of Medical Sciences. This is an open-access article distributed under the terms of the Creative Commons Attribution License (http://creativecommons.org/licenses/by/4.0), which permits unrestricted use, distribution, and reproduction in any medium, provided the original work is properly cited.
} 
Table 1. Histogenetic Classification of Benign and Malignant Tumors

\begin{tabular}{lll}
\hline Normal Tissue & Benign Tumors & Malignant Tumors \\
\hline Glandular epithelium & Adenoma & Adenocarcinoma \\
Surface/ squamous epithelium & Papilloma & Squamous carcinoma \\
Fibroblasts & Fibroma & Fibrosarcoma \\
Cartilage & Chondroma & Chondrosarcoma \\
Striated muscle & Rhabdomyoma & Rhabdomyosarcoma \\
Smooth muscle & Leiomyoma & Leiomyosarcoma \\
Blood vessels & Haemangioma & - \\
Endothelium & - & Angiosarcoma \\
Fat & Lipoma & Liposarcoma \\
Bone & Osteoma & Osteosarcoma \\
Liver & Hepatoma & Hepatocellular carcinoma \\
\hline
\end{tabular}

depending on their site.

\section{Genetic Factors in Cancer \\ Cancer Genes}

Cancer is a multifactorial disease and it can be hereditary ( $10 \%$ to $15 \%$ of cases) where defect resides in every single cell of the body as it passes from parents to offspring through gametes. However, $85 \%-90 \%$ of cancer cases are sporadic in nature, which happen by chance and carry alterations only in descendents of peripheral blood cells, without any familial clustering. Cancer is a complicated biological process that involves alterations of many genes regulating various functions, such as cell growth and its motility, cell death, extra cellular matrix remodeling, detoxification of carcinogen compounds, and DNA repair. Genes responsible for cancer phenotype are mainly divided into two major classes: high penetrance genes and low penetrance genes. In high penetrance genes, oncogenes (growth promoting), apoptosis genes, and tumor suppressor genes (growth suppressing) are involved whereas in low penetrance genes, drug metabolising genes/cancer susceptibility genes are involved. High penetrance genes mainly play a role in progression of cancer whereas low penetrance genes are mainly involved in the susceptibility of the disease..$^{3-5}$

\section{Oncogenes}

Proto-oncogenes are normal cellular components involved in regulating cell proliferation and differentiation. According to their function, proto-oncogenes are classified into growth factors, growth factor receptors, signal transducers, cell cycle transducers, cell cycle regulators, and transcription factors. Mutations in proto-oncogenes like point mutations, translocation and gene amplification deregulate their expression and/or alter their structure, and turn them into a tumor inducing agent. These defects are known as gain of function. More than 100 oncogenes have been identified so far. ${ }^{6}$ Many proto-oncogenes are most frequently activated in various cancers. ${ }^{7}$ Some genetic changes play a crucial role in the development of some neoplasms namely over-expression, ampli- fication, and oncogene mutations in c-Ki-ras, HER2/neu (aka C-erb-B2), and c-myc. ${ }^{8,9}$

\section{Tumor Suppressor Genes}

The first tumor suppressor gene, the RB (retinoblastoma), was identified in 1986. Genetic or epigenetic changes such as deletions, abnormal splicing, point mutations, promoter methylation, deregulation of imprinting and haplo-insufficiency can either inactivate or regulate tumor suppressor genes. ${ }^{10}$ Tumor suppressor genes are negative regulators of cell growth and are called gatekeepers. They are also involved in cell differentiation and programmed cell death. They are classified as cell cycle regulators, transcriptional regulators, or signaling proteins. Methylation, cytogenetic aberrations, loss of heterozygosity, genetic mutations, gain of auto inhibitory function, and polymorphism either abolish or reduce the function of tumor suppressor genes and therefore they are targeted by loss of function in tumor cells. Abnormalities of the tumor-suppressor TP53 (alias p53) and adenomatous polyposis coli $(A P C)$ genes are found in over $60 \%$ and $50 \%$ of cancer patients, respectively. ${ }^{8,10,11}$

\section{Low Penetrance Genes}

Various cancers have major variations in incidence. This fact indicates a multi-factorial etiology in cancers which constitutes environmental factors, socio-cultural, genetic as well as ethnic backgrounds. In cancer, genetic predisposition may result from alterations in several low penetrance genes. It means that single mutations are far less common contributors in genetic predisposition for cancer. Genetically predisposed individuals might develop non-hereditary, sporadic cancers in which several low penetrance genes but not single mutations are responsible. The Human Genome Project has explored the single nucleotide polymorphisms (SNPs) and other polymorphisms like deletions, variable number of tandem repeats (VNTR), and gene amplifications as low penetrance genes which may lead to inter-individual differences in susceptibility and genetic predisposition to cancer. ${ }^{3,4}$

There are classical genetic approaches for identifying sus- 
ceptibility genes for familial cancers; however, they are not very successful in identifying genes for sporadic cancers. Sporadic cancers are considered to be polygenic and multi-factorial diseases in which individual differences in susceptibilities are likely to depend not only on the function of whole series of low penetrance genes but also on their interaction with environmental factors. Therefore, understanding cancer biology involves not only the study of high penetrance genes, but also the identification of low penetrance genes which can modify cancer risk. ${ }^{12,13}$

\section{Ethical Approval}

Not applicable.

\section{Competing Interests}

Authors declare that they have no competing interests.

\section{Acknowledgments}

We sincerely acknowledge the financial support of Department of Biotechnology, Government of India (grant No. BT/PR7270/MED/ 30/909/2012).

\section{References}

1. Taunton J. How to starve a tumor. Chem Biol. 1997;4:493-6.

2. Campbell K. The Genetic Basis of Cancer. London: Nursing Time; 2000.

3. Houlston RS, Peto J. The search for low-penetrance cancer susceptibility alleles. Oncogene. 2004;23(38):6471-6. doi: 10.1038/sj.onc. 1207951.

4. Imyanitov EN, Togo AV, Hanson KP. Searching for cancer-associated gene polymorphisms: promises and obstacles. Cancer Lett. 2004;204:3-14. doi: 10.1016/j. canlet.2003.09.026.

5. Farzaneh F, Roberts S, Mandal D, et al. The IL-10 -1082G polymorphism is associated with clearance of HPV infection. BJOG. 2006;113(8):961-4. doi: 10.1111/j.14710528.2006.00956.x.

6. Krontiris TG. Oncogenes: Molecular Medicine. New England Journal of Medicine 1995;333:303-6. doi: 10.1056/ nejm199508033330508.

7. Wang LD, Zheng S, Liu B, et al. CYP1A1 GSTs and $\mathrm{mEH}$ polymorphism and susceptibility to esophageal carcinoma:study of population from a high-incidence area in north China. World J Gasteronterol. 2003;9:1394-7. doi: 10.3748/wjg.v9.i7.1394.

8. Charles D. Cancer Management: A Multidisciplinary Approach. 11th ed. 2008.

9. Sarasin A, Kauffmann A. Overexpression of DNA repair genes is associated with metastasis: a new hypothesis. Mutat Res. 2008;659(1-2):49-55. doi: 10.1016/j. mrrev.2007.12.002.

10. Kuroki T, Trapasso F, Shirainshi T, Alder h, Mimori K, Mori M. Croce CM, Genetic alterations of the tumor suppressor gene WWOX in esophageal squamous cell carcinoma. Cancer Res. 2002;62:2258-60.

11. Knudson AG. Two genetic hits (more or less) to cancer. Nat Rev Cancer. 2001;1:157-62.

12. Houlston RS, Tomlinson IP. Detecting low penetrance genes in cancer: the way ahead. J Med Genet. 2000;37(3):161-7. doi: 10.1136/jmg.37.3.161.

13. te Meerman GJ, de Vries EG. Relevance of high and low penetrance. Lancet. 2001 Jul 28;358(9278):331-2. doi: 10.1016/S0140-6736(01)05506-4. 